\title{
OPTIMAL SIZING AND PLACEMENT OF DISTRIBUTED GENERATORS AND CAPACITORS IN RADIAL DISTRIBUTION NETWORK
}

\author{
Partha P Biswas ${ }^{1}$, P. N. Suganthan ${ }^{1}$ and Gehan A J Amaratunga ${ }^{2}$ \\ ${ }^{1}$ School of Electrical \& Electronic Engg., \\ Nanyang Technological University, Singapore \\ ${ }^{2}$ Department of Engg., University of Cambridge, UK
}

\begin{abstract}
Distribution network is an integral part of power system and it acts as the interface with consumer load points. The network incurs significant amount of losses in the electrical system. Loss minimization remains one of the prime objectives in distribution network optimization. This paper proposes an application of L-SHADE algorithm to simultaneously size and place both distributed generators (DGs) and shunt capacitors (SCs) in distribution network to reduce system real power loss. SHADE is the success history based parameter adaptation technique of differential evolution (DE). L-SHADE improves the performance of SHADE by linearly reducing the population size in successive generations. The algorithm is applied to minimize loss in standard IEEE 33-bus radial distribution network (RDN) and the simulation results are compared with some recent studies on the topic.
\end{abstract}

\section{KEYWORDS}

Radial distribution network, Distributed generator $(D G)$, Shunt capacitor (SC), Power loss, LSHADE algorithm

\section{INTRODUCTION}

Mitigating ever increasing load demand is one of the major challenges faced by utility companies. It may not always be feasible to boost capacity of transmission network. Locally installed distributed generators (DG) and added shunt capacitor (SC) banks in the system can augment the capacity, reduce losses, improve voltage profile and power quality of the network. Distributed generators can be a diesel generator, a wind turbine, solar photovoltaics (PV), fuel cells etc. Optimal sizing and siting of both distributed generators and shunt capacitors are of significance in improving system performance. Several literatures [1-3] focused on optimal sizing and placement of DGs only in pursuit of real power loss minimization. A network comprising both DG and SC has been studied in few literatures. Naik et al. [4] took analytical approach to optimally size and locate both the components. In most recent papers, heuristic methods such as hybrid harmony search algorithm (HSA) and particle artificial bee colony (PABC) [5], intersect mutation differential evolution (IMDE) []] and back-tracking search algorithm (BSA) [7] have been applied for optimal design of capacity and placement of both distributed generators and shunt 
capacitors. Ref. [ $[$ ] optimizes size and location of both DG and SC in various networks with simultaneous minimization of both real and reactive power losses using decomposition based multi-objective evolutionary algorithm (MOEA/D).

The current study implements L-SHADE [9] algorithm to optimally locate and size both DG and SC in radial distribution network. SHADE [10]is a success history based parameter adaptation technique of DE optimization process for a constrained, multimodal non-linear problem. The convergence of the algorithm to global optima is fast and it potentially outperforms most other DE variants on CEC benchmark problems [9]. SHADE exhibited good performance in optimal power flow solutions [11]. In recent times L-SHADE has successfully been applied and has shown very competitive performance in windfarm layout optimization [12], in total harmonic distortion minimization of multilevel inverters [13], in hybrid active power filter parameter optimization [14] etc. Motivated by the growing application and noteworthy performance of LSHADE in power domain, we apply the algorithm on the problem of radial distribution network (RDN). The distribution network is to be reinforced with optimally sized and appropriately placed DGs and SCs so that network real power loss is minimized. As an obvious fact, the problem is about simultaneous optimization of discrete variables i.e. locations (bus nos.) of all the components and continuous variables i.e. ratings of all the components. Further, the problem is non-linear due to the requirement of power flow calculation that involves numerous system components.

The organization of rest of the paper is done following way. Section 2 includes the mathematical formulation of power flow in distribution network. Section 3 describes the algorithm and its application. Section 4 discusses the case studies and simulation results. The paper ends with conclusion and possible future work in section 5 .

\section{MATHEMATICAL MODEL}

The mathematical formula pertaining to power flow in the network are presented in this section with the aid of a simple feeder configuration.

\subsection{Power flow formulation}

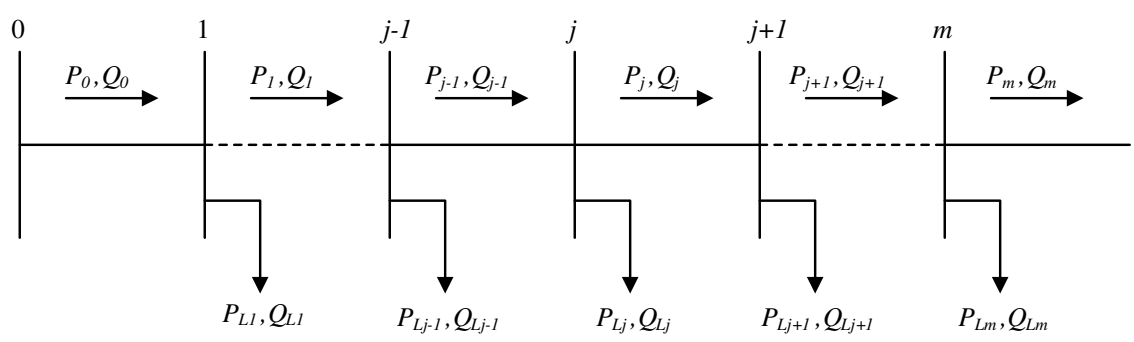

Fig. 1. Single line diagram of a radial feeder [ $[\underline{]}]$

Single line diagram of a simple feeder-line configuration is shown in Fig. 1. The computation of power flow is performed by following equations [8]:

$$
P_{j+1}=P_{j}-P_{L j+1}-R_{j, j+1} \cdot \frac{P_{j}^{2}+Q_{j}^{2}}{\left|V_{j}\right|^{2}}
$$




$$
\begin{gathered}
Q_{j+1}=Q_{j}-Q_{L j+1}-X_{j, j+1} \cdot \frac{P_{j}^{2}+Q_{j}^{2}}{\left|V_{j}\right|^{2}} \\
\left|V_{j+1}\right|^{2}=\left|V_{j}\right|^{2}-2\left(R_{j, j+1} \cdot P_{j}+X_{j, j+1} \cdot Q_{j}\right) \\
+\left(R_{j, j+1}{ }^{2}+X_{j, j+1}{ }^{2}\right) \cdot \frac{P_{j}^{2}+Q_{j}^{2}}{\left|V_{j}\right|^{2}}
\end{gathered}
$$

where, real power and reactive power flowing out of bus $j$ are $P_{j}$ and $Q_{j}$ respectively; $P_{L j+1}$ and $Q_{L j+1}$ are the real load and reactive load connected at bus $j+1$. Line section between buses $j$ and $j+1$ has resistance $R_{j, j+1}$ and reactance $X_{j, j+1} .\left|V_{j}\right|$ is the voltage magnitude of bus $j$. The power loss in line segment connecting buses $j$ and $j+1$ is computed using following equation:

$$
P_{L o s s}(j, j+1)=R_{j, j+1} \cdot \frac{P_{j}^{2}+Q_{j}^{2}}{\left|V_{j}\right|^{2}}
$$

where, real power loss is defined by $P_{\text {Loss }}$. Total real power loss, the optimization objective in the network, is obtained by summing up all the line losses as follows:

$$
T P_{\text {Loss }}=\sum_{j=0}^{m-1} P_{\text {Loss }}(j, j+1)
$$

In this study, we consider DG units supplying real power with unity power factor e.g. photovoltaic systems, micro turbines etc. Therefore, if a DG, delivering power output of $P_{D G}$, is added to a bus (say $j$-th bus), the load in that bus changes from $P_{L j}$ to $\left(P_{L j}-P_{D G}\right)$. Similarly, if $k$-th bus in the system has inductive load of $Q_{k}$, an addition of $Q_{C}$ unit of capacitor bank alters the reactive load to $\left(Q_{k}-Q_{C}\right)$. During the search process, the algorithm checks all possible locations with all probable ratings of the equipment to decide best combination that results in minimum power loss.

\subsection{Constraints}

Magnitude of any bus voltage must lie within specified limits of maximum and minimum voltages. Current in any branch shall not exceed the rated capacity of the branch. Mathematically, these can be written as:

$$
\begin{aligned}
& V_{\min } \leq\left|V_{j}\right| \leq V_{\max } \\
& \left|I_{j, j+1}\right| \leq I_{j, j+1(\max )}
\end{aligned}
$$

where, $V_{\max }$ and $V_{\min }$ are the maximum and minimum allowable voltages for any bus in the network. The numerical values of these parameters for the systems are considered as 0.90 p.u. and 1.05 p.u. respectively. $\left|I_{j, j+1}\right|$ is the magnitude of current flowing in the line linking bus $j$ and bus $j+1$, while $I_{j, j+1 \text { (max) }}$ is the maximum permissible current through the same branch considering the thermal capability limit of the line. It is worthwhile to mention that current carrying capacities of the branches in IEEE bus system are not explicit. Moreover, as installation of DGs and SCs improve the voltage profile of the network, the current reduces from the base configuration. Hence, verification of this constraint is not necessary. 


\section{L-SHADE ALGORITHM AND APPLICATION}

Differential Evolution (DE) is a stochastic, population based optimization algorithm [15]. SHADE [10] is success history based adaptive DE where control parameters scaling factor $(F)$ and crossover rate $(C R)$ are automatically adjusted during the evolution process. Algorithm LSHADE [9] is an extension of SHADE. In L-SHADE, the population size is dynamic and it reduces in successive generations following a linear function. The algorithm alongwith its applicationon RDN problem is briefly described in this section.

\subsection{Initialization}

Firstly, DE optimization process creates an initial population of probable solutions by assigning random values (within feasible bound) to each decision vector of the population. Initialization of $j$-th component of $i$-th decision vector is done as [16]:

$$
x_{i, j}^{(0)}=x_{\min , j}+\operatorname{rand}_{i j}[0,1] *\left(x_{\max , j}-x_{\min , j}\right)
$$

where $\operatorname{rand}_{i j}[0,1]$ is a random number lying between 0 and 1 . The superscript ' 0 ' signifies initialization of population members.

\subsection{Mutation}

In next step during mutation process, donor/mutant vector $v_{i}^{(t)}$ is created corresponding to each population member or target vector $x_{i}^{(t)}$ in the current generation $t$. The mutation strategy used here is 'current-to-pbest/1':

$$
v_{i}^{(t)}=x_{i}^{(t)}+F_{i}^{(t)} \cdot\left(x_{\text {pbest }}^{(t)}-x_{i}^{(t)}\right)+F_{i}^{(t)} \cdot\left(x_{R_{1}^{i}}^{(t)}-x_{R_{2}^{i}}^{(t)}\right)
$$

The mutually exclusive integers $R_{1}^{i} \& R_{2}^{i}$ are randomly chosen from the population range [1, $\left.N p\right]$; $x_{p b e s t}^{(t)}$ is randomly selected from top $100 p \%(p \in[0,1])$ individuals of current generation. The positive control parameter $F_{i}^{(t)}$ scales the difference vectors at $t$-th generation. The mutation strategy adopted in L-SHADE helps to exploit the search space efficiently and converge into an optimal solution.

\subsection{Parameter adaptation}

At each generation $t$, each individual has its own $F_{i}^{(t)}$ and $C R_{i}^{(t)}$ parameters that are used to generate the trial vector. Adaptation of these two parameters follows as:

$$
\begin{aligned}
F_{i}^{(t)} & =\operatorname{randc}\left(\mu F_{r}^{(t)}, 0.1\right) \\
C R_{i}^{(t)} & =\operatorname{randn}\left(\mu C R_{r}^{(t)}, 0.1\right)
\end{aligned}
$$

where $\operatorname{randn}\left(\mu C R_{r}^{(t)}, 0.1\right)$ and $\operatorname{randc}\left(\mu F_{r}^{(t)}, 0.1\right)$ are the sampled values from Normal and Cauchy distributions respectively. Normal distribution has a mean of $\mu C R_{r}^{(t)}$ and a variance of 0.1 . Location and scale parameters of Cauchy distribution are $\mu F_{r}^{(t)}$ and 0.1 respectively. $\mu F_{r}^{(t)} \& \mu C R_{r}^{(t)}$ are randomly chosen from successful candidates of past generations saved in a 
memory. The two values are initialized to 0.5 and subsequently modified by weighted Lehmer mean $[9,10]$.

\subsection{Crossover}

Donor vector $v_{i}^{(t)}$ enters into the trial/offspring vector $u_{i}^{(t)}=\left(u_{i, 1}^{(t)}, u_{i, 2}^{(t)}, \ldots ., u_{i, d}^{(t)}\right)$ by mixing its components with target vector $x_{i}^{(t)}$ through crossover. Binomial crossover is most commonly employed and it operates on each element based on adapted crossover rate $C R_{i}^{(t)}$. The scheme for an element is defined as:

$$
u_{i, j}^{(t)}=\left\{\begin{array}{c}
v_{i, j}^{(t)} \text { if } j=j_{\text {rand }} \text { orrand }_{i, j}[0,1] \leq C R_{i}^{(t)} \\
x_{i, j}^{(t)} \text { otherwise }
\end{array}\right.
$$

where $j_{\text {rand }}$ is a randomly chosen natural number in $\{1,2, \ldots, d\}$, and $d$ is the dimension of the decision vector.

\subsection{Linear population size reduction}

The success of SHADE algorithm is attributed to the adaptation technique of scale factor $F$ and crossover rate $C R$.It has also been found that dynamic reduction in population size improves performance of SHADE. L-SHADE precisely implements the task by introducing a linear function for reduction of population size in successive generations. The population size starts with $N p_{\text {ini }}$ (initial population size) and reduces closely matching the linear function before finally ending with $N p_{\min }$ (minimum population size).After each generation $t$, the population size in subsequent generation $t+1$ is calculated by -

$$
N p(t+1)=\operatorname{round}\left[\left(\frac{N p_{\min }-N p_{\text {ini }}}{N F E_{\max }}\right) \cdot N F E+N p_{\text {ini }}\right]
$$

$N p_{\min }$ is set to 4 because mutation strategy adopted here requires minimum 4 individuals. $N F E_{\text {max }}$ is the maximum number of fitness evaluationsand $N F E$ is the current number of fitness evaluations. If $N p(t+1)<N p(t)$, worst ranking individuals totalling $[N p(t)-N p(t+1)]$ are removed from the population [9]. A summary of steps involved in the optimization process is provided herein.

\section{A. Input and initialization:}

1. Input $N p_{\text {ini }}=100, N F E_{\max }=20000$.

2. Define vector $x=[$ position, rating $]$ and range of all these elements. We have 2 DG and 2 SC to size and allocate. So, a maximum of 4 elements for position (bus no.) and 4 elements for rating will form vector $x$ (maximum, $d=8$ ). Elements of position will always be rounded off to nearest integer.

3. Create random initial population of 100 such vectors defined as $x_{i}$ as per equation (8), $i=$ $1,2, \ldots 100$.

4. Set generation counter $t=0$, dynamic population size $N p(t)=N p_{\text {ini }}$, evaluation counter $N F E=1$ and control parameters $\mu F_{r}^{(0)}=\mu C R_{r}^{(0)}=0.5$.

B. Algorithm loop: 
1. Evaluate $f\left(x_{i}^{(t)}\right)$ i.e. ' $T P_{\text {Loss }}$ ' in equation (5) for $x_{i}^{(t)}$ where $i=1$ to $N p$. Increase counter $N F E$ by $N p$ i.e. $N F E=N F E+N p$.

2. while termination criteria $N F E<N F E_{\max }$ do

3. for $i=1$ to $N p$ do

4. Adapt control parameters $F_{i}^{(t)}$ and $C R_{i}^{(t)}$ as per equations (10) \& (11).

5. Perform mutation to generate vector $v_{i}^{(t)}$ as per equation (9).

6. Perform crossover to generate element $u_{i, j}^{(t)}$ as per equation (12).

7. Evaluate $f\left(u_{i}^{(t)}\right)$ i.e. 'TP $P_{\text {Loss }}$ ' for $u_{i}^{(t)}$. Increase evaluation counter $N F E$ by 1 i.e. $N F E=$ NFE+1.

8. Select best fit individuals for next generation. If, $f\left(\mathrm{u}_{i}{ }^{(t)}\right) \leq f\left(x_{i}^{(t)}\right)$ and constraints in eq. (6) \& (7) are satisfied, $x_{i}^{(t+1)}=u_{i}^{(t)}$. Else $x_{i}^{(t+1)}=x_{i}^{(t)}$.

End for loop.

9. Update population size for next generation $N p(t+1)$ as per LPSR strategy in equation (13).

10. Increase generation counter $t=t+1$. Go to step 2 of algorithm loop.

\section{CASE STUdY RESUltS AND COMPARISON}

IEEE 33-bus standard radial distribution network (RDN) diagram is provided in Fig. 2. Total load of the network is $3.72 \mathrm{MW}$ and 2.3 MVAr. The maximum cumulative capacities of DGs (unity power factor) and capacitor banks in the installation are not to exceed $2 \mathrm{MW}$ and $2 \mathrm{MVAr}$ respectively. Table 1 summarizes the case description, results and comparison. 3 case studies are performed for the RDN. Case-1 deals with addition of only DGs in the network. Case-2 is study of the RDN when only SCs are added, while Case-3 considers both DGs and SCs. Average runtime in normal PC for most complex Case-3 is about 90 seconds for one complete run of the algorithm (i.e. $N F E_{\max }=20000$ function evaluations) on MATLAB platform. As can be seen from the tabulated results, increasing number of DG and SC reduces the system loss drastically. However, as in [6], we consider maximum 2 nos. of DG and 2 nos. of SCs that can be connected to the network. The loss data given by L-SHADE algorithm for all cases are the lowest. In Case1, bus location and equipment ratings proposed by L-SHADE, IMDE and BSA are quite similar. However, bus-13 for allocation of one DG is preferred to bus-14 for effectiveness in loss reduction. In Case-2, cumulative ratings of SCs suggested by both IMDE and L-SHADE are almost equal. Again, bus-12 is advantageous location for one SC rather than bus-14. Ratings proposed by L-SHADE for SCs are higher in Case-3 when compared with IMDE algorithm. However, resulting network loss, the prime objective of optimization, is much reduced with little reshuffle in placement of both DG and SC. 


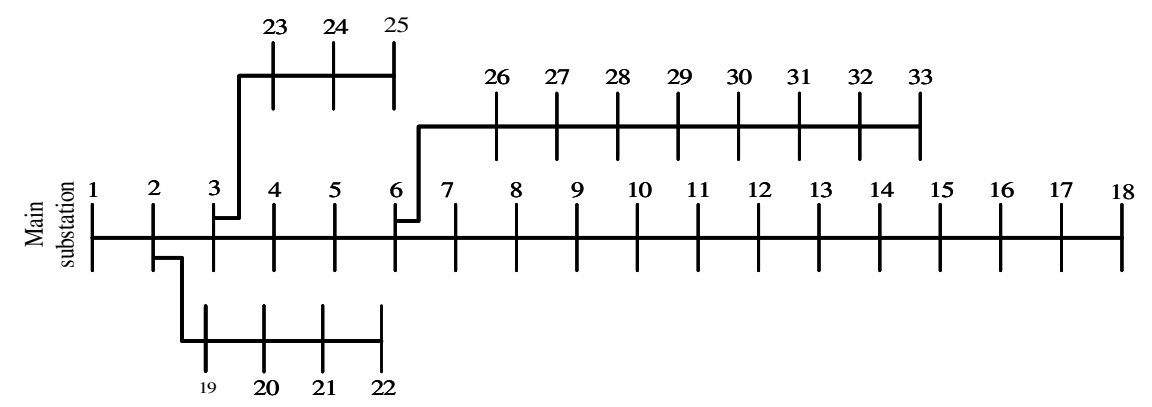

Fig. 2: Standard IEEE-33 bus test system

Table 1. Simulation results for IEEE 33-bus radial distribution network

\begin{tabular}{|c|c|c|c|c|c|c|}
\hline \multirow{2}{*}{$\begin{array}{l}\text { Case } \\
\text { description }\end{array}$} & \multirow[t]{2}{*}{ Parameter } & \multicolumn{5}{|c|}{ Various optimization methods } \\
\hline & & L-SHADE & IMDE [ㅁ] & Analytical [4] & Hybrid [5] & $\mathrm{BSA}[\underline{7}]$ \\
\hline \multirow{3}{*}{$\begin{array}{l}\text { Case-1 } \\
\text { (DG only) }\end{array}$} & Realpower loss, kW & 85.91 & $86.12^{1}$ & 142.34 & 111.03 & 87.16 \\
\hline & $\begin{array}{l}\text { DG size, kW } \\
\text { (busno.) }\end{array}$ & $\begin{array}{l}85(13), \\
1155(30)\end{array}$ & $\begin{array}{l}840(14) \\
1130(30)\end{array}$ & $1000(18)$ & $2598(6)$ & $\begin{array}{l}851.6(13), \\
1157.6(30)\end{array}$ \\
\hline & $\begin{array}{l}\text { Min bus voltage in } \\
\text { p.u. (bus no.) }\end{array}$ & $0.9685(33)$ & $0.9675^{1}(33)$ & $0.9311(33)$ & $0.9425(18)$ & not reported \\
\hline \multirow{3}{*}{$\begin{array}{l}\text { Case-2 } \\
\text { (SC only) }\end{array}$} & Realpower loss, kW & 135.75 & 139.7 & 164.6 & & - \\
\hline & $\begin{array}{l}\text { SC size, kVAr } \\
\text { (bus no.) }\end{array}$ & $\begin{array}{l}467(12), \\
1039(30)\end{array}$ & $\begin{array}{l}475(14), \\
1037(30)\end{array}$ & $1000(33)$ & & - \\
\hline & $\begin{array}{l}\text { Min bus voltage in } \\
\text { p.u. (bus no.) }\end{array}$ & $0.9360(18)$ & $0.942(18)$ & $0.9165(18)$ & & - \\
\hline \multirow{4}{*}{$\begin{array}{l}\text { Case-3 } \\
(\mathrm{DG}+\mathrm{SC})\end{array}$} & Real power loss, kW & 28.50 & 32.08 & 84.28 & 58.45 & 30.87 \\
\hline & $\begin{array}{l}\text { DG size, kW } \\
\text { (bus no.) }\end{array}$ & $\begin{array}{l}829(13) \\
1121(30)\end{array}$ & $\begin{array}{l}1080(10), \\
896.4(31)\end{array}$ & $\begin{array}{l}447(18), \\
559(17)\end{array}$ & $2531(6)$ & $\begin{array}{l}860(13), \\
1310.5(30)\end{array}$ \\
\hline & $\begin{array}{l}\text { SC size, kVAr } \\
\text { (bus no.) }\end{array}$ & $\begin{array}{l}452(12), \\
1041(30)\end{array}$ & $\begin{array}{l}254.8(16), \\
932.3(30)\end{array}$ & $\begin{array}{l}400(33), \\
500(32)\end{array}$ & $1250(30)$ & $\begin{array}{l}334.8(14), \\
899.8(30)\end{array}$ \\
\hline & $\begin{array}{l}\text { Min bus voltage in } \\
\text { p.u. (bus no.) }\end{array}$ & $0.9803(25)$ & $0.979(25)$ & $0.961(30)$ & $0.9536(18)$ & not reported \\
\hline
\end{tabular}

${ }^{1}$ Values are recalculated with the proposed ratings of DG and SC []]

Fig. 3 shows convergence of L-SHADE for Case-3. As observed from the diagram, the algorithm converges in less than 8000 fitness evaluations. Linear population size reduction technique is demonstrated in Fig. 4 where the population size reduces almost linearly to 4 individuals. Fig. 5 indicates bus voltage profiles for various case studies performed in this literature. Voltages of all buses are within the allowable limits. Further, more uniform voltage profile is obtained when multiple components with smaller ratings are distributed throughout the network. 


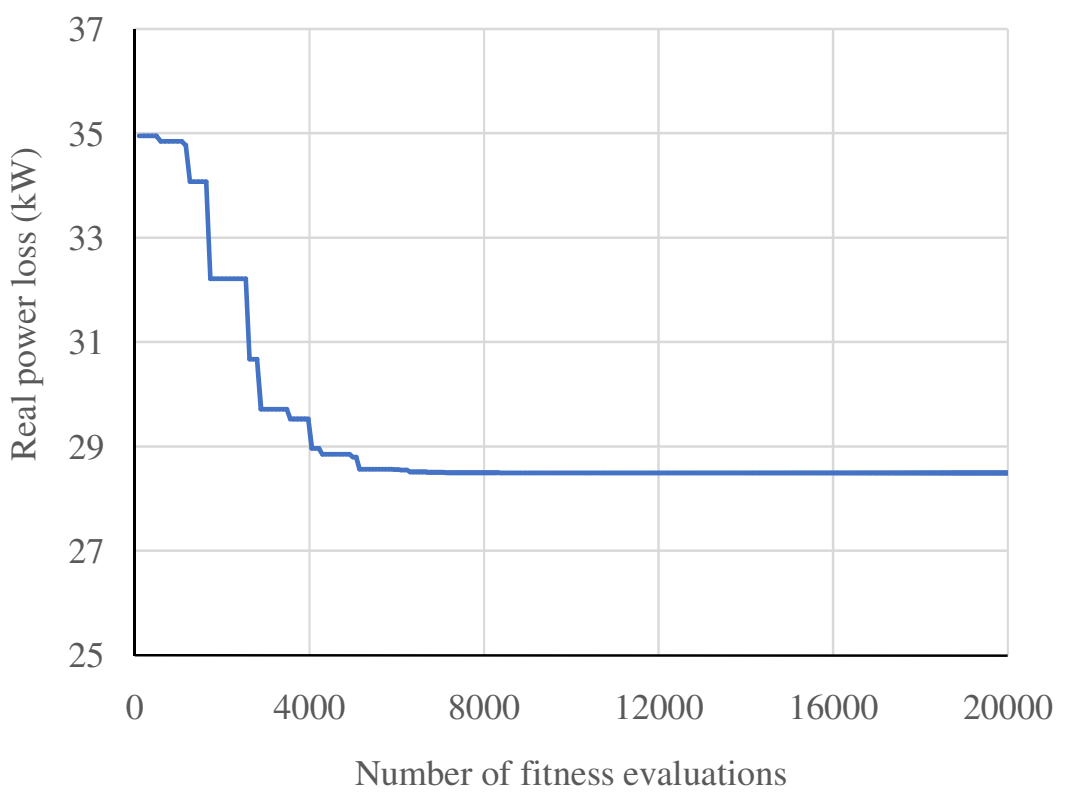

Fig. 3: Convergence of L-SHADE algorithm for Case-3

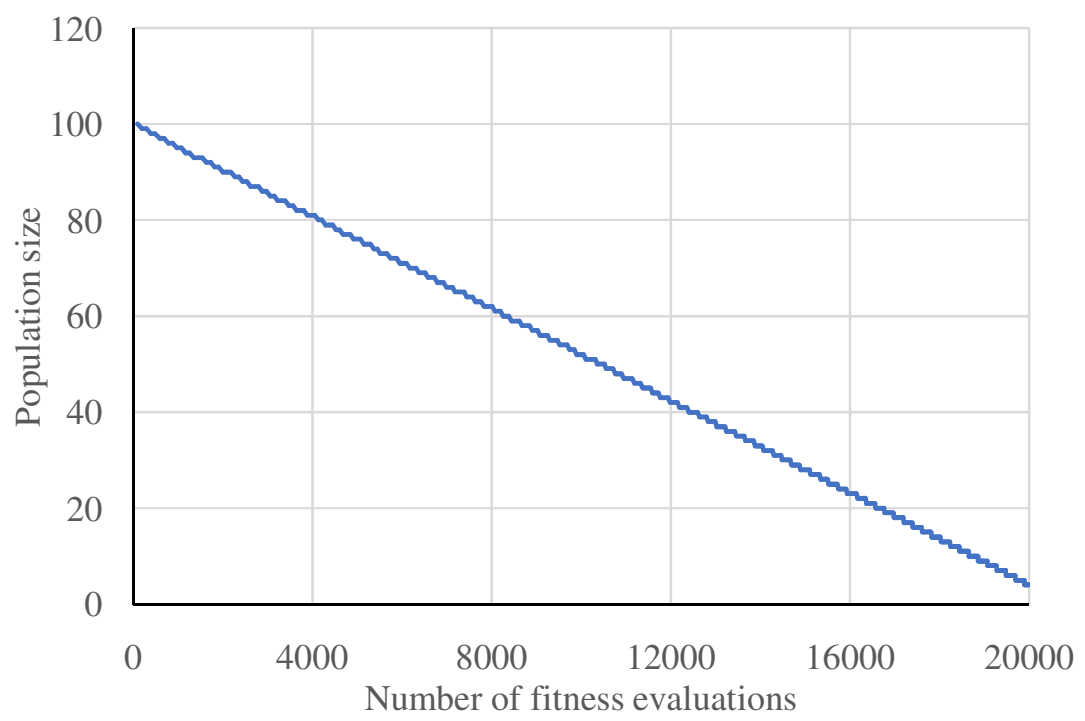

Fig. 4: Linear population size reduction of L-SHADE algorithm for Case-3 


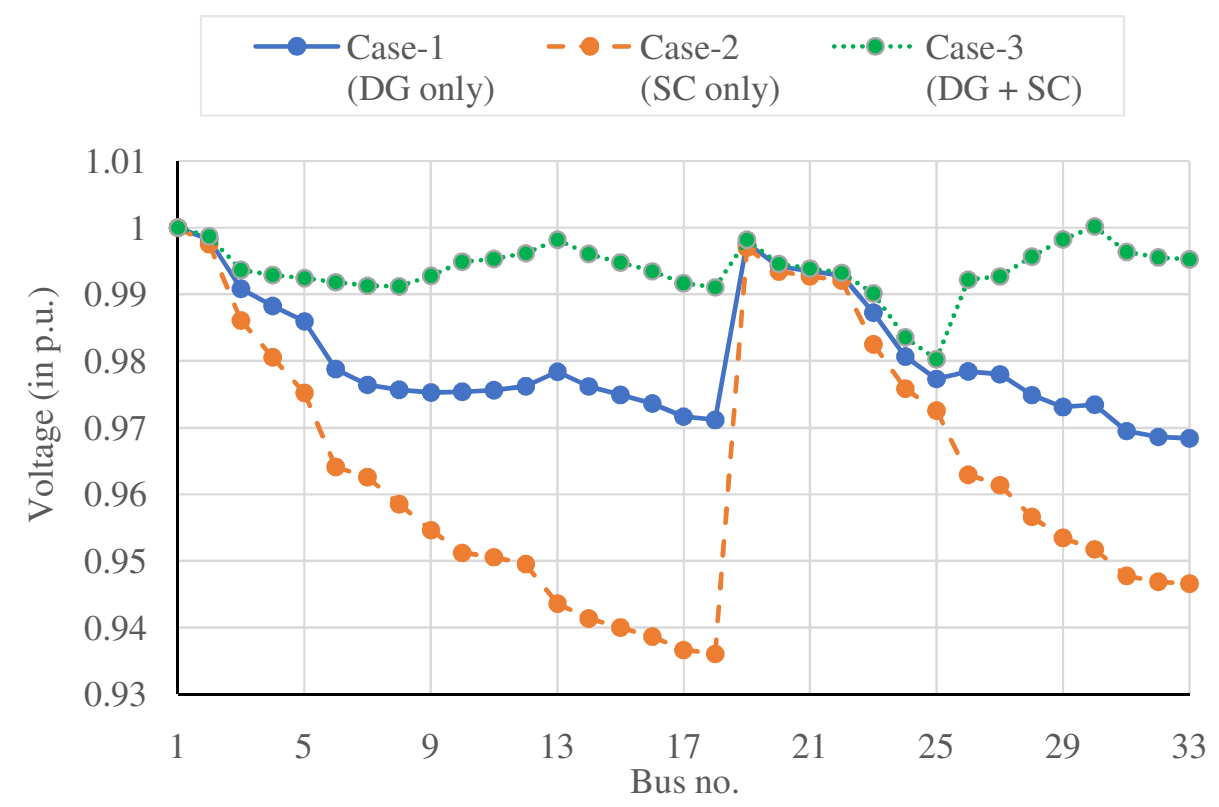

Fig. 5: Bus voltage profile of 33-bus system for various case studies

\section{CONCLUSION}

The present paper discusses in detail the application and usefulness of L-SHADE optimization algorithm for deciding the rating and location of DG and SC simultaneously in the distribution network to reduce real (active) power loss. Size and location proposed by the algorithm for the equipment lead to lower system real power loss than the loss achieved by other equivalent algorithms. Reduction of loss by any amount is of technical and commercial advantage. Further the algorithm converges to the optimal solution very fast. The effectiveness of the algorithm in reducing loss in the networks with large number of buses remains the topic for future study.

\section{ACKNOWLEDGEMENTS}

This project is funded by the National Research Foundation Singapore under its Campus for Research Excellence and Technological Enterprise (CREATE) program.

\section{REFERENCES}

[1] Viral, R., \& Khatod, D. K. (2015). An analytical approach for sizing and siting of DGs in balanced radial distribution networks for loss minimization. International Journal of Electrical Power \& Energy Systems, 67, 191-201.

[2] Ayodele, T. R., Ogunjuyigbe, A. S. O., \& Akinola, O. O. (2015). Optimal location, sizing, and appropriate technology selection of distributed generators for minimizing power loss using genetic algorithm. Journal of Renewable Energy, 2015.

[3] Karimyan, P., Gharehpetian, G. B., Abedi, M., \& Gavili, A. (2014). Long term scheduling for optimal allocation and sizing of DG unit considering load variations and DG type. International Journal of Electrical Power \& Energy Systems, 54, 277-287.

[4] Naik, S. G., Khatod, D. K., \& Sharma, M. P. (2013). Optimal allocation of combined DG and capacitor for real power loss minimization in distribution networks. International Journal of Electrical Power \& Energy Systems, 53, 967-973. 
[5] Muthukumar, K., \& Jayalalitha, S. (2016). Optimal placement and sizing of distributed generators and shunt capacitors for power loss minimization in radial distribution networks using hybrid heuristic search optimization technique. International Journal of Electrical Power \& Energy Systems, 78, 299319.

[6] Khodabakhshian, A., \& Andishgar, M. H. (2016). Simultaneous placement and sizing of DGs and shunt capacitors in distribution systems by using IMDE algorithm. International Journal of Electrical Power \& Energy Systems, 82, 599-607.

[7] Fadel, W., Kilic, U., \& Taskin, S. (2016). Placement of Dg, Cb, and Tcsc in radial distribution system for power loss minimization using back-tracking search algorithm. Electrical Engineering, 1-12.

[8] Biswas, P. P., Mallipeddi, R., Suganthan, P. N., \& Amaratunga, G. A. (2017). A multiobjective approach for optimal placement and sizing of distributed generators and capacitors in distribution network. Applied Soft Computing.

[9] Tanabe, R., \& Fukunaga, A. S. (2014, July). Improving the search performance of SHADE using linear population size reduction. In Evolutionary Computation (CEC), 2014 IEEE Congress on (pp. 1658-1665). IEEE.

[10] Tanabe, R., \& Fukunaga, A. (2013, June). Success-history based parameter adaptation for differential evolution. In Evolutionary Computation (CEC), 2013 IEEE Congress on (pp. 71-78). IEEE.

[11] Biswas, P. P., Suganthan, P. N., \& Amaratunga, G. A. (2017). Optimal power flow solutions incorporating stochastic wind and solar power. Energy Conversion and Management, 148, 1194-1207.

[12] Biswas, P. P., Suganthan, P. N., \& Amaratunga, G. A. (2017, June). Optimal placement of wind turbines in a windfarm using L-SHADE algorithm. In Evolutionary Computation (CEC), 2017 IEEE Congress on (pp. 83-88). IEEE.

[13] Biswas, P. P., Awad, N. H., Suganthan, P. N., Ali, M. Z., \& Amaratunga, G. A. (2017, June). Minimizing THD of multilevel inverters with optimal values of DC voltages and switching angles using LSHADE-EpSin algorithm. In Evolutionary Computation (CEC), 2017 IEEE Congress on (pp. 77-82). IEEE.

[14] Biswas, P. P., Suganthan, P. N., \& Amaratunga, G. A. (2017). Minimizing Harmonic Distortion in Power System with Optimal Design of Hybrid Active Power Filter using Differential Evolution. Applied Soft Computing.

[15] Qin, A. K., Huang, V. L., \& Suganthan, P. N. (2009). Differential evolution algorithm with strategy adaptation for global numerical optimization. IEEE transactions on Evolutionary Computation, 13(2), 398-417.

[16] Biswas, P. P., Suganthan, P. N., \& Amaratunga, G. A. (2017). Optimization of Wind Turbine Rotor Diameters and Hub Heights in a Windfarm Using Differential Evolution Algorithm. In Proceedings of Sixth International Conference on Soft Computing for Problem Solving (pp. 131-141). Springer, Singapore. 


\section{AUTHORS}

\section{Partha P. Biswas}

Partha P. Biswas received his Bachelor of Electrical Engineering degree from Jadavpur University, Kolkata, India in the year 2002. He was awarded MSc in Power Engineering from Nanyang Technological University, Singapore in 2016. He has worked in different engineering companies at various positions for about 13 years in Singapore and India. Currently he is pursuing his Ph.D. in the School of Electrical and Electronic Engineering, Nanyang Technological University. His research topic is optimization of power system using evolutionary algorithms.

\section{Ponnuthurai N. Suganthan}

Ponnuthurai N. Suganthan receivedthe B.A. degree, the Postgraduate Certificate, andthe M.A. degree in electrical and informationengineering from the University of Cambridge,U.K., in 1990, 1992, and 1994respectively. He received the Ph.D. degree inelectrical and electronic engineering from the Schoolof Electrical and Electronic Engineering, NanyangTechnological University, Singapore in 1996. Heis currently an Associate Professor in the School of Electrical andElectronic Engineering, Nanyang Technological University. His research interests include evolutionarycomputation, applications of evolutionary computation, neural networks, pattern recognition and bioinformatics.

\section{Gehan A. J. Amaratunga}

Gehan A. J. Amaratunga received the B.S. degree in electrical engineering from Cardiff University, Wales, U.K., and the Ph.D. degree in electrical engineering from University of Cambridge, U.K. He has been holding the 1966 Professorship in Engineering at the University of Cambridge since 1998, where he is currently the Head of the Electronics, Power, and Energy Conversion Group, Electrical Engineering Division, Department of Engineering. He has worked for 20 years on integrated and discrete electronic devices for power conversion and on the science and technology of carbon-based electronics for 15 years. He also leads an active research effort in novel solar-cell technologies. Dr. Amaratunga is a Fellow of the
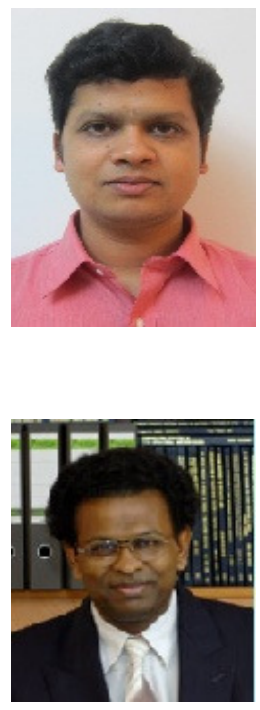
Royal Academy of Engineering, U.K

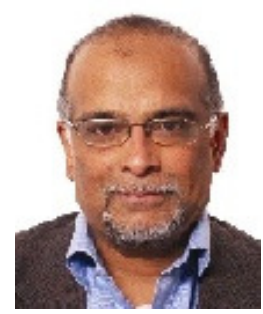

